\title{
Transductive-Weighted Neuro-fuzzy Inference System for Tool Wear Prediction in a Turning Process
}

\author{
Agustín Gajate ${ }^{1}$, Rodolfo E. Haber ${ }^{1}$, José R. Alique ${ }^{1}$, and Pastora I. Vega ${ }^{2}$ \\ ${ }^{1}$ Instituto de Automática Industrial, Spanish Council for Scientific Research, \\ Ctra. Campo Real Km. 0.200, Arganda del Rey, 28500 Madrid, Spain \\ \{agajate, rhaber, jralique\} @iai.csic.es \\ ${ }^{2}$ Departamento de Informática y Automática, Universidad de Salamanca, \\ Pza. de los Caídos s/n, 37008 Salamanca, Spain \\ pvega@usal.es
}

\begin{abstract}
This paper presents the application to the modeling of a novel technique of artificial intelligence. Through a transductive learning process, a neuro-fuzzy inference system enables to create a different model for each input to the system at issue. The model was created from a given number of known data with similar features to data input. The sum of these individual models yields greater accuracy to the general model because it takes into account the particularities of each input. To demonstrate the benefits of this kind of modeling, this system is applied to the tool wear modeling for turning process.
\end{abstract}

Keywords: Transductive reasoning, Neuro-fuzzy inference system, Modeling, Tool wear.

\section{Introduction}

Nowadays, new strategies for modeling dynamic systems have become essential to qualitatively improve complex and large-scale processes. A model is considered to be a depiction of the physical properties of an object or process, so if we have a model of the process, we actually have a tool for planning, recognition and depiction of the information involved in the process. Moreover, we can infer properties from the process that can be used to understand, to predict and to control the process itself.

There are two ways of dealing with process modeling. The first approach ("white box") is based on the assumption that the process can be fully described by mathematical equations representing the corresponding physical laws [1]. The second approach ("black box") is based on the idea that the process is completely unknown and there is no a priori knowledge of the model structure to reflect the process's physical behavior. The unknown model parameters are estimated using experimental data to obtain an input/output relationship. The main advantage of the "black box" approach when using accurately measured data is that it is possible to develop a model without requiring physical process knowledge [2]. The main drawbacks of this method lie in the structure of the model, which is unable to offer any physical meaning. In practice, the best way is to combine the two approaches, if possible, so that the more thoroughly-known parts can be modelled using physical knowledge and the less-known 
ones can be approximated through the "black box" approach. This is what is known as the "gray box" approach [3].

Sometimes the process is extremely complex and fraught with uncertainty, and its behavior is practically impossible to describe exactly by conventional modelling tools. In that case approaches based on artificial intelligence techniques like Fuzzy Logic and Neural Networks are the best way to cope with this problem. Recent years have been characterized by the development of new paradigms in the field of artificial intelligence (AI). Nowadays, the hybridization of fuzzy logic with neural networks is the most well-established and best-known method.

By the late nineties, several hybrid neuro-fuzzy systems have already been developed, which may be separated into two major groups: neural networks endowed with the ability to handle fuzzy information [fuzzy-neural networks (FNN)] [4,5], and fuzzy systems combined with neural networks in order to enhance certain desirable characteristics [neural-fuzzy systems (NFS)] [6,7]. A deep review of available neuralfuzzy strategies goes beyond the scope of the paper [8,9].

The simplest and easiest way to obtain a neuro-fuzzy model is to create its knowledge base using verbalization techniques. Frequently, a complete verbal description of how a complex process behaves is quite difficult to obtain. In such situations an identification procedure is required. So then, a neuro-fuzzy model can be built from measured input/output ("black box") data using engineering knowledge about the process variables, goals and disturbances ("white box") by applying a recursive identification technique.

From the viewpoint of systems theory and system modeling, transductive methods generate a model at a single point of the workspace. For each new datum that has to be processed, the closest examples are selected among the known data, with the goal of creating a new local model that dynamically approximates the process in its new state as close as possible. The main issue is therefore how to assign more weight to the specific information related with the datum to be processed than to the general information provided by the entire training set [10].

Machining processes are widely used in manufacturing. Four basic types of operations are turning, drilling, milling, and grinding, performed by different machine tools. Indeed, the importance of maximising a tool's working time and doing the utmost to keep tools from breaking is directly related with turning-process optimisation. The key issue is to find an appropriate trade-off between tool wear and productivity considering the tool's cost, its replacement cost, the cost of writing off the machine's idle time, and so forth. Avoiding breakage derived from a excessive tool wear is another capital factor, because replacing the tool after it breaks means increased costs, since the post-breakage stage is one of the trickiest, most unpredictable times, aside from the damage that may be done to the part and, not unusually, to the whole machine itself.

From the best of authors' knowledge, transductive reasoning methods have not been applied yet for modeling of machining processes. Moreover, this work proposes the application of a Transductive-Weighted Neuro-Fuzzy Inference System to obtain local models for predicting tool wear in a turning process [11]. Furthermore, the proposed strategy for modeling is simpler, faster and more accurate than other neuro-fuzzy inference systems to deal with complex processes. The main contributions are therefore not only a modified strategy of an state-of-the-art neuro-fuzzy inference system for modeling, but also its application for monitoring tool wear in a turning process. 


\section{Neuro-fuzzy Inference Systems and Transductive Reasoning}

In terms of learning procedures, most of evolutionary neuro-fuzzy strategies apply inductive reasoning systems. In inductive reasoning the key issues is to find a general model drawn from the entire set of input/output data representing the whole system. The model is later used for designing the required control system. In contrast, there are transductive reasoning methods that generate a model at a single point of the workspace. Transductive methods have some advantages over inductive methods, because sometimes creating a valid model for the entire space or region of operation is a difficult task, yielding insufficient performance in some cases. The dynamic generation of local models enables to easily expand the represented knowledge as the set of known data facilitating incremental on-line learning. In addition, these strategies are capable of functioning correctly with a small training set.

Transductive reasoning methods have been applied to text recognition applications [12], time series prediction and medical diagnosis applications [11]. However, on the basis of reviewed literature, applications in the field of manufacturing processes have not been previously reported.

Neuro-fuzzy inference techniques combine the paradigms of fuzzy logic and neural networks in order to take advantage of both techniques achieving the simplicity of modelling (neural networks), while providing knowledge explicitly expressed in a set of "if-then" rules (fuzzy logic).

\subsection{Transductive-Weighted Neuro-fuzzy Inference System}

This work is inspired on a new paradigm of neuro-fuzzy inference systems to obtain local models of the process. Transductive-Weighted Neuro-Fuzzy Inference System (TWNFIS) is a relatively new transductive reasoning system that consists in a dynamic neuro-fuzzy inference system with local generalization [11]. TWNFIS is endowed with three important characteristics: Neural, Fuzzy and Trasductive.

- Neural: Excellent ability to model any nonlinear function with a high accuracy in addition to possessing a high learning capacity.

- Fuzzy: Semantic transparency, ability to represent human thought as well as excellent behavior before uncertainty and imprecision.

- Transductive: Estimation of the model in a single input/output set of the space, using only information related with the corresponding set.

The relevant steps for modeling on the basis of TWNFIS are given as follows. The system's inputs can be treated in different kinds of physics units but the normalization is recommended. In this paper, each input data $x^{\prime}$ is normalized according to (1):

$$
x=\frac{x^{\prime}-\mu_{x}}{\sigma_{x}}
$$

where $\mu_{x}$ is the mean and $\sigma_{x}$ is the standard deviation of the set of known data or training set. 
A local model is created using data from the training set that are the closest to each new input datum. The weighted Euclidean distance is used for selecting each data subset (2). The size of the subset $\left(N_{q}\right)$ is one parameter of the algorithm. Weights $\left(w_{j}\right)$ of each element of the input vector $\left(w_{j} \in[0,1]\right)$ are computed in a posteriori modeladjusting process, reflecting the importance of each variable. Initially they all have unitary value.

$$
\|\bar{x}-\bar{y}\|=\left[\frac{1}{P} \sum_{j=1}^{P} w_{j}\left|x_{j}-y_{j}\right|^{2}\right]^{\frac{1}{2}}
$$

where $P$ is the number of elements in the input data vector, $\bar{x}$ is the input data vector, and $\bar{y}$ is each one of the vectors in the training set.

Membership functions are built iteratively on the basis of the closest data. TWNFIS uses the Evolving Clustering Method (ECM) to create these functions. The main difference with regard to the approach here-in proposed is the use of a clustering algorithm more suitable for real-time modelling dynamic systems instead of ECM. A clustering strategy called Quality Cluster Algorithm (QT_Clust) is then applied [13]. This algorithm utilizes two parameters: a threshold to indicate a maximum diameter of the clusters and a minimum number of elements (data) in a cluster. A candidate cluster is created using the first datum. The other elements are iteratively added without exceeding the maximum diameter. A second candidate cluster is formed starting with the second data and repeating the procedure. The number of candidate clusters is equal to the number of closest data. At this point, the largest candidate cluster is selected and retained. Data are removed from consideration and the entire procedure is repeated on the smaller set. The resulting clusters are ellipsoids. The center and the radio of the clusters set the center and the width of the Gaussian membership functions, respectively.

The weight of each variable is adjusted according to its relevance within each subspace. A gradient-descent algorithm to optimize the weights and parameters of the fuzzy rules is then applied. If the closest neighbors do not change due to the new adjusted weights of the variables, a new model is created setting the weights obtained in the previous iteration. Finally, the model is used to predict the system output.

Defuzzification is done using a modified center of area method. The resulting error function is stated as a weighted quadratic error function that is derivable:

$$
E=\frac{1}{2} v_{i}\left[f\left(x_{i}\right)-d_{i}\right]^{2}
$$

where $f\left(x_{i}\right)$ is the defuzzification function that yields the output, $d_{i}$ are the target values, and $v_{i}$ indicates the proximity of each target to the expected prediction. A Gradient-descent algorithm is then applied to after deriving (3).

\subsection{Tool Wear Model in Turning Process}

Tool wear is generally caused by a combination of various phenomena, although it is an event inherent to the cutting process. Tool wear can occur gradually or in drastic 
breakdowns. Gradual wear may occur by adhesion, abrasion or diffusion, and it may appear in two ways: wear on the tool's face or wear on its flank. Contact with the chip produces a crater into the tool's face. Flank wear, on the other hand, is commonly due to friction between the tool and the work piece material.

Tool wear is not a physical variable whose value may be measured by any specific method, but rather a subjective estimate a specialist can make, depending on the condition of the tool's edges and surfaces. Since there is no single criterion for deciding when a tool needs sharpening, different lifetimes may be predicted for the same tool employed in the same process. Two widely used criteria are catastrophic failure and changes in tool geometry. Other criteria that are sometimes used are a degraded toolsurface finish, deviation in cutting forces, increased power consumption, overheating, non-tolerant pieces and the appearance of chattering. This paper deals the tool wear modeling of the turning process. Data supplied by [14] coming from the measurement of different sensors such as acoustic emissions signals, vibrations (accelerations) and cutting forces (Fig. 1). Therefore, it is a multiple-input/single-output model to predict tool wear.

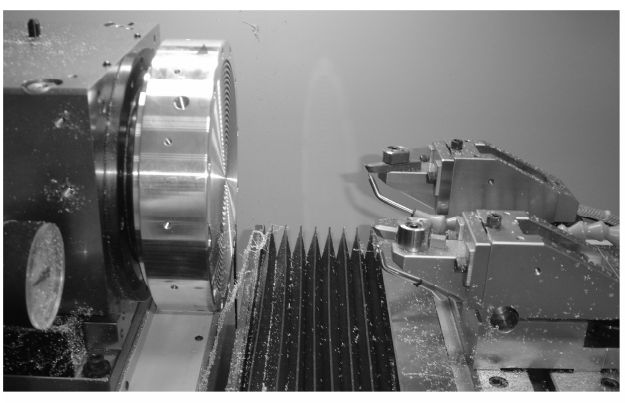

Fig. 1. Turning process and sensors related to the model

The choice of cutting force, vibration and acoustic emission signals is because they are the most affected by the tool wear. Among the cutting force signals, it has become experimentally that the cutting force in the $z$ axis is more sensitive to changes in the wear compared to the cutting forces in the components on the $x$ and $y$ axes.

\section{Results}

Modeling of tool wear for turning processes is based on the experimental data provided in [14]. For the sake of simplicity the table with experimental data is shown in Appendix I and a brief description is given as follows.

The experimental study was carried out for turning operations on cast iron work piece material and uncoated coated carbide insert tool material The process parameters were: cutting speed $(94 \mathrm{~m} / \mathrm{min}$ and $188 \mathrm{~m} / \mathrm{min})$, feed: $0.06 \mathrm{~mm} / \mathrm{rev}$ and 0.08 $\mathrm{mm} / \mathrm{rev}$, depth: $0.7 \mathrm{~mm}$ (constant). The turning operations were carried out on a high precision lathe machine. Acoustic emission signals (ring down count), vibrations (acceleration), cutting forces and tool wear were recorded for each operation on the 
machine. The authors stated that tool wear (flank wear) was measured off-line using a microscope.

The tool wear $T_{w}{ }^{\prime}$ was modeled through the time $t$, the cutting force in the direction of the cutting speed $F_{z}$, the vibrations (accelerations) of the tool $a_{t}$, and the acoustic emission signals $A E S$. Therefore, depending on the model, the tool wear was estimated as follows:

$$
T_{w}^{\prime}=\hat{H}\left(t, F_{z}, a_{t}, A E S\right)
$$

where $\hat{H}$ represents the corresponding neuro-fuzzy system.

The Total Average Error TAE (5) is used to assess the accuracy of the model. Additionally, another measure of accuracy is the number of data that cross an individual error of $10 \%$.

$$
T A E=\frac{1}{n} \sum_{n} \frac{\left|T_{w}-T_{w}{ }^{\prime}\right|}{T_{w}} \cdot 100
$$

where $T_{w}$ is the real tool wear, $T_{w}{ }^{\prime}$ is the modeled tool wear and $n$ is the number of data of each experiment.

The parameters of the transductive neuro-fuzzy inference system that best match the model with the process after testing several configurations correspond to: three neighbors in the algorithm, clustering threshold value of 2.53 (maximum diameter) and one as a minimum number of elements. The parameters of TWNFIS and inductive neuro-fuzzy inference system (ANFIS) [14] for modeling tool wear are shown in Table 1.

The results obtained by both models are shown in Fig. 2 and Table 2. TWNFIS outperformed ANFIS in the first and second experiment whereas the results of applying these techniques are very similar for the third experiment. Only in the fourth experiment ANFIS showed better accuracy than TWNFIS.

Table 1. Neuro-fuzzy algorithms for modeling tool wear

\begin{tabular}{lll}
\hline Algorithm & Inductive System [14] & Transductive System \\
\hline System & MISO & MISO \\
Clustering & Substractive & Quality Algorithm \\
Membership functions type & Gaussian & Gaussian \\
Inference system & Takagi-Sugeno & Mamdani \\
Number of membership functions & 7 & Variable each run (max. 3) \\
Number of rules & 7 & Variable each run (max. 3) \\
Training algorithms & BP + Least Square Method & Back Propagation \\
Iterations & 3 & 3 \\
Learning rate & $10^{-3}$ & $10^{-3}$ \\
Error tolerance & 0 & 0 \\
Training data set & 24 samples & 24 samples \\
Validation data set & 47 samples & 47 samples \\
\hline
\end{tabular}




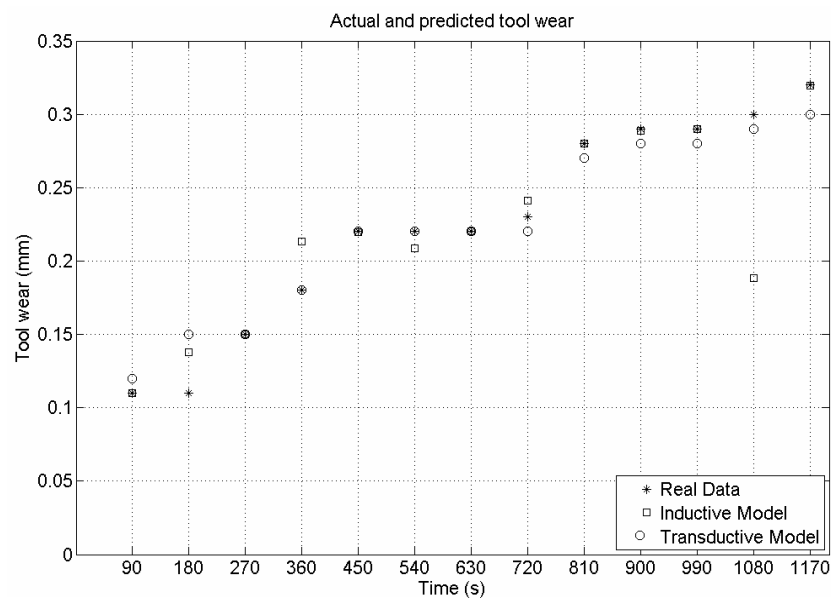

Fig. 2. Real tool wear and obtained with the models for experiment 1

Table 2. Average errors of the created models

\begin{tabular}{ccccc}
\hline Experiment & Inductive Model [14] & Point with AE $>10 \%$ & Proposed Model & Point with AE $>10 \%$ \\
\hline 1 & $7.12 \%$ & 3 & $5.37 \%$ & 1 \\
2 & $40.4 \%$ & 4 & $5.30 \%$ & 3 \\
3 & $3.46 \%$ & 1 & $3.98 \%$ & 0 \\
4 & $1.97 \%$ & 0 & $7.19 \%$ & 3 \\
\hline
\end{tabular}

\section{Discussion}

The first application of a paradigm for modeling a complex dynamical process inspired in TWNFIS is proposed in this paper. The introduction of a more efficient clustering technique to this strategy in order to deal with computing and real-time constraints is a novelty with regard to the original approach proposed in [11].

Likewise, from the best of authors knowledge, the application for modeling tool wear in a real manufacturing process (a turning process), has not been previously reported in the literature. The comparison of this technique with ANFIS according with the work reported in [14] demonstrated the superiority of the suggested approach.

From technical viewpoint an efficient computational model for predicting tool wear is essential. The importance of maximising a tool's working time and doing the utmost to keep tools from breaking is directly related with cutting-process optimisation. The key issue is to find an appropriate trade-off between tool wear and productivity considering the tool's cost, its replacement cost, the cost of writing off the machine's idle time, and so forth.

Acknowledgments. This work was supported by DPI2008-01978 COGNETCON and CIT-420000-2008-13 NANOCUT-INT projects of the Spanish Ministry of Science and Innovation. 


\section{References}

1. Ljung, L.: System Identification: Theory for the User. Prentice-Hall, Upper Saddle River (1999)

2. Sjoberg, J., Zhang, Q., Ljung, L., Benveniste, A., Delyon, B., Glorennec, P.Y., Hjalmarsson, H., Juditsky, A.: Nonlinear black-box modeling in system identification: a unified overview. Automatica, 1691-1724 (1995)

3. Bohlin, T.: A Case-Study of Gray Box Identification. Automatica, 307-318 (1994)

4. Keller, J.M., Hunt, D.J.: Incorporating fuzzy membership functions into the perceptron algorithm. IEEE Transactions on Pattern Analysis and Machine Intelligence, PAMI 7, 693-699 (1985)

5. Mitra, S., Hayashi, Y.: Neuro-fuzzy rule generation: survey in soft computing framework. IEEE Transactions on Neural Networks 11, 748-768 (2000)

6. Jang, J.-S.R.: ANFIS: adaptive-network-based fuzzy inference system. IEEE Transactions on Systems, Man and Cybernetics 23, 665-685 (1993)

7. Kim, J., Kasabov, N.: HyFIS: Adaptive neuro-fuzzy inference systems and their application to nonlinear dynamical systems. Neural Networks 12, 1301-1319 (1999)

8. Nauck, D., Klawonn, F., Kruse, R.: Foundations of Neuro-Fuzzy Systems. Wiley, Chichester (1997)

9. Wang, L.X.: A course in fuzzy systems and control. Prentice-Hall, Inc., Upper Saddle River (1996)

10. Vapnik, V.: Statistical Learning Theory. John Wiley \& Sons, Inc., New York (1998)

11. Song, Q., Kasabov, N.: TWNFI - a transductive neuro-fuzzy inference system with weighted data normalization for personalized modeling. Neural Networks 19, 1591-1596 (2006)

12. Joachims, T.: Transductive inference for text classification using support vector machines. In: Machine Learning: Proceedings of the Sixteenth International Conference (1999)

13. Heyer, L.J., Kruglyak, S., Yooseph, S.: Exploring expression data identification and analysis of coexpressed genes. Genome Research 9, 1106-1115 (1999)

14. Sharma, V.S., Sharma, S.K., Sharma, A.K.: Cutting tool wear estimation for turning. Journal of Intelligent Manufacturing 19, 99-108 (2008) 